\title{
Lodo de esgoto tratado na composição de substrato para produção de mudas de Plathymenia reticulata Benth
}

\author{
Sewage sludge treated in the substrate composition for Plathymenia reticulata \\ Benth seedling production
}

\author{
David Pessanha Siqueira', Deborah Guerra Barroso ${ }^{\mathrm{II}}$, \\ Giovanna Campos Mamede Weiss de Carvalho ${ }^{\text {III }}$, Rommel Monnerat Erthal ${ }^{\text {IV }}$, \\ Maria Clara Coutinho Rodrigues ${ }^{\mathrm{v}}$, Claudio Roberto Marciano ${ }^{\mathrm{VI}}$
}

\begin{abstract}
Resumo
O trabalho teve como objetivo avaliar o uso do lodo de esgoto tratado (LET) associado a substrato à base de casca de pinus (SC1) ou à base de fibra de coco (SC2), em diferentes proporções, como substrato para produção de mudas de vinhático. O experimento foi conduzido em casa de vegetação, localizada na unidade de apoio a pesquisa da Universidade Estadual do Norte Fluminense, de junho a novembro de 2016. Utilizaram-se 20,40,60, 80\% de LET associados ao SC1 ou ao SC2, 100\% de LET e o tratamento com SC1 com adubação convencional (controle). O lodo de esgoto foi higienizado com adição de cal a 15\% do peso seco. Os resultados revelam que as menores proporções de LET no substrato resultaram em mudas com melhor crescimento em altura, diâmetro do coleto, área foliar, massa seca da parte aérea e do sistema radicular, comprimento e volume radicular. A nodulação foi negativamente influenciada nas mudas produzidas em proporções acima de $20 \%$ de LET. A agregação do torrão ao substrato não foi influenciada pela adição de LET, entretanto, houve aumento da curvatura do torrão nas mudas produzidas em maiores quantidades de LET. O conteúdo da parte aérea de $\mathrm{N}, \mathrm{P}$ e $\mathrm{K}$ foi menor nas mudas produzidas em substrato com LET. Para produção de mudas de vinhático, sugere-se a proporção de $20 \%$ de lodo de esgoto associado ao SC1 ou SC2, não havendo prejuízos em relação às mudas produzidas em substrato comercial adubado, utilizado comercialmente.
\end{abstract}

Palavras-chave: Vinhático; Substratos alternativos; Biossólido; Qualidade do torrão

\begin{abstract}
The aim of this work was to evaluate the treated sewage sludge (LET) associated with commercial substrate based on pinus bark (SC1) or based on coconut fiber (SC2), in different proportions, as substrate for the production of vinhático seedlings. The study was led in a greenhouse, located in the research support unit of the State University of North Fluminense. The treatments considered as substrates were 20, 40 , $60,80 \%$ of LET associated with SC1 or SC2, 100\% of LET and the treatment with SC1 with conventional fertilization (control). The sewage sludge was sanitized with addition of lime to $15 \%$ of dry weight. The results showed that the lower LET proportions in the substrate resulted in seedlings with better growth in height, stem diameter, leaf area, dry mass of shoot and root system, root length and volume. The nodulation was negatively influenced in the seedlings produced in proportions above $20 \%$ LET. The aggregation of the clod was not influenced by the addition of LET, however, there was increase of the curvature of the clod in the seedlings produced in larger amounts of LET. The shoot content of N, P and K was lower in the seedlings with LET. For the production of vinatic seedlings, $20 \%$ of sewage sludge associated with SC1 or SC2, is suggested, with no losses in relation to the seedlings produced in commercial substrate that received fertilization, used commercially.

Keywords: Vinhático; Alternative substrates; Biossolid; Clod quality

\footnotetext{
Engenheiro Agrônomo, MSc., Doutorando pelo Programa de Pós-Graduação em Produção Vegetal, Universidade Estadual do Norte Fluminense, Av. Alberto Lamego, CEP 28013-602, Campos dos Goytacazes (RJ), Brasil. dps@pq.uenf.br (ORCID: 0000-0002-0756-0153).

Engenheira Agrônoma, Drª ., Professora Associada do Laboratório de Fitotecnia, Universidade Estadual do Norte Fluminense, Av. Alberto Lamego, CEP 28013-602, Campos dos Goytacazes (RJ), Brasil. deborahbarroso@gmail.com (ORCID: 0000-0002-6869-8076) Alberto Lamego, CEP 28013-602, Campos dos Goytacazes (RJ), Brasil. giovannacampos85@yahoo.com.br (ORCID: 0000-0002-6896-2359) erthalrm@gmail.com (ORCID: 0000-0003-3399-645X)

Engenheira Agrônoma, Universidade Estadual do Norte Fluminense, Av. Almberto Lamego, CEP 28013-602, Campos dos Goytacazes (RJ), Brasil. mariaclaracoutinho3@gmail.com (ORCID: 0000-0001-8222-4582) Alberto Lamego, CEP 28013-602, Campos dos Goytacazes (RJ), Brasil. marciano@uenf.br (ORCID: 0000-0002-5344-6087)
}

III Engenheira Agrônoma, Mestranda pelo Programa de Pós-Graduação em Produção Vegetal, Universidade Estadual do Norte Fluminense, Av.

Iv Graduando(a) em Agronomia, Universidade Estadual do Norte Fluminense, Av. Alberto Lamego, CEP 28013-602, Campos dos Goytacazes (RJ), Brasil.

vı Engenheiro Agrônomo, Dr., Professor Associado do Laboratório de Solos, Universidade Estadual do Norte Fluminense, Av.
\end{abstract}




\section{Introdução}

A espécie florestal Plathymenia reticulata Benth, conhecida como vinhático, pertence à família das Fabaceae e pode ser considerada pioneira ou tardia, dependendo do bioma que se localiza. É uma espécie adaptada a terrenos pobres, com potencial para atender à demanda atual de mudas para recuperação de áreas degradadas e adequação das propriedades rurais. Além disso, possui madeira de boa qualidade, com aptidão para exploração comercial (LACERDA et al., 2002; CARVALHO, 2010).

As exigências de medidas ambientais compensatórias, bem como a exploração das florestas nativas são fatores que contribuem para o aumento da demanda de mudas nativas para recuperação das áreas, revegetação, e adequação ambiental das propriedades rurais (CALDEIRA et al. 2013; DELARMELINA et al., 2014). Neste cenário, a composição do substrato é um fator de grande influência sobre as mudas que serão ofertadas ao mercado, devendo propiciar a formação de mudas com qualidade satisfatória, facilitando as operações do plantio e garantindo bom desempenho pós-plantio. Além das questões ambientais, nos últimos anos houve aumento da demanda de mudas nativas também para atender à silvicultura econômica.

O processo de tratamento dos esgotos para retorno da água aos corpos hídricos gera um resíduo denominado de lodo de esgoto que é um resíduo de caráter orgânico e que apresenta teores de componentes orgânicos e inorgânicos. A maior parte deste resíduo é destinada aos aterros sanitários, sendo um procedimento complexo e dispendioso, buscando-se alternativas para uma destinação mais adequada, entre as quais se destaca o uso agrícola, em especial no setor florestal (GOMES et al., 2013). O lodo de esgoto, após tratamento para uso agrícola, pode também ser denominado de biossólido.

A destinação do lodo de esgoto para o setor florestal é promissora, destacando-se a utilização na formulação de substrato para produção de mudas. Embora o lodo apresente elevados teores de matéria orgânica e nutrientes, e ainda beneficie a retenção de água, deve-se ponderar a quantidade adicionada deste resíduo na formulação final do substrato, pela possibilidade de possuírem substâncias prejudiciais às plantas, assim, na maioria das vezes, não deve ser utilizado como substrato puro (ROCHA et al., 2013; SANTOS et al., 2014; TRAZZI et al., 2014; SIQUEIRA et al., 2018).

Pesquisas relacionadas ao efeito de diferentes composições de substrato sobre a qualidade de mudas formadas são constantes e atuais, buscando-se integrar os preceitos da sustentabilidade, proporcionar diminuição do tempo e dos custos do processo produtivo, além de fornecer opções para os viveiristas. Desta forma, este estudo teve como objetivo avaliar o uso de lodo de esgoto tratado na composição de substrato para produção de mudas de Plathymenia reticulata Benth, puro ou associado a substratos comerciais à base de casca de pinus ou fibra de coco, avaliando a qualidade das mudas formadas e do torrão.

\section{Material e métodos}

O experimento foi realizado em casa de vegetação, localizada na unidade de apoio à pesquisa da Universidade Estadual do Norte Fluminense, no Município de Campos dos Goytacazes - RJ (UAP - UENF), durante o período de junho a novembro de 2016. A temperatura média para o período experimental foi de $24,23^{\circ} \mathrm{C}$ e a umidade relativa média de $72,51 \%$. A casa de vegetação conta com sistema de irrigação automático via microaspersão com lâmina de água aplicada de $4 \mathrm{~mm} \mathrm{dia}^{-1}$, parcelada três vezes ao dia.

Para formulação dos substratos que compunham os tratamentos foram utilizadas as seguintes proporções de lodo de esgoto tratado (LET): 20, 40, 60 e 80\% associado ao substrato comercial à base de casca de pinus Basaplant Florestal ${ }^{\circledR}$ (SC1) ou ao substrato comercial à base de fibra de coco Golden Mix ${ }^{\circledR}$ textura fina (SC2) - (v:v). Além desses, utilizaram-se 100\% de LET e 100\% do SC1, no qual foi adicionado $8 \mathrm{~kg}$ de adubo de liberação lenta (15-9-12 N-P-K) por $\mathrm{m}^{3}$ de substrato, considerado como tratamento controle. 
O lodo de esgoto utilizado foi cedido pela empresa "Águas do Paraíba”, prestadora de serviços do Município de Campos dos Goytacazes - RJ, produzido na estação de tratamento da Chatuba, cujos teores de metais pesados observados e os limites estabelecidos pela legislação vigente para destinação agrícola (BRASIL, 2006) estão descritos na Tabela 1, estando o lote apto para tal finalidade. Para higienização do lodo, foi adicionado cal (BRASIL, 2006) a 15\% do peso seco (LOUSADA, 2015).

Tabela 1 - Teores de metais pesados do lote de lodo de esgoto doado pela "Águas do Paraíba" e os requisitos mínimos para destinação agrícola, conforme a resolução do $\mathrm{N}^{\circ} 375 / 2006$ do CONAMA.

Table 1 - Content of heavy metals in sewage sludge donated by “Águas do Paraíba” and minimum requirements for agricultural destination, according to CONAMA resolution No. $375 / 2006$.

\begin{tabular}{lcc}
\hline \multirow{2}{*}{ Parâmetros } & Resultados analíticos & Resolução n $^{\circ}$ \\
\cline { 2 - 3 } & & 375/2006 CONAMA \\
\hline Arsênio & 2,96 & $\mathbf{~ m g ~ k g}^{-1}$ \\
Bário & 202,75 & 1300 \\
Cádmio & 0,79 & 39 \\
Chumbo & 31,84 & 300 \\
Cobre & 205,09 & 1500 \\
Cromo & 65,26 & 1000 \\
Mercúrio & 0,19 & 17 \\
Molibdênio & 8,75 & 50 \\
Níquel & 31,31 & 420 \\
Selênio & 2,56 & 100 \\
Zinco & 846,32 & 2800 \\
\hline
\end{tabular}

Determinação feita pelo traço dos elementos em água ou resíduo (Método 3050B), realizada pela empresa Hidroquímica - Grupo Oceanus.

As sementes de vinhático utilizadas para produção das mudas foram coletadas em novembro de 2015, no Município de Trajano de Moraes - RJ, obtidas de três matrizes. As mudas foram então produzidas por semeadura direta em tubetes de polipropileno de $180 \mathrm{~cm}^{3}$, já contendo as diferentes composições de substrato, sendo semeadas duas sementes por tubete e o raleio feito quando as mudas apresentavam entre 3 e $5 \mathrm{~cm}$, deixando-se a mais centralizada e vigorosa.

O experimento foi instalado em blocos casualizados, com quatro blocos e 20 mudas por parcela, totalizando 80 mudas úteis para cada tratamento. Antes da semeadura, foi realizada a análise física do substrato, determinando-se a densidade de partículas, do substrato e a porosidade total (Tabela 2), pelo método do balão volumétrico (EMBRAPA, 2017). 
Tabela 2 - Densidade de partícula, densidade do substrato e porosidade total das diferentes proporções de lodo de esgoto tratado (LET) em mistura com o substrato à base de casca de pinus (SC1) ou substrato à base de fibra de coco (SC2), utilizados na produção de mudas de Plathymenia reticulata Benth.

Table 2 - Particle density, substrate density and total porosity of different proportions of sewage sludge treated (LET) with substrate based on pinus bark (SC1) or substrate based on coconut fiber (SC2) in the production of Plathymenia reticulate Benth seedlings.

\begin{tabular}{|c|c|c|c|}
\hline Tratamento & $\begin{array}{c}\text { Densidade partícula } \\
\mathrm{g} \mathrm{cm}^{-3} \\
\end{array}$ & $\begin{array}{c}\text { Densidade substrato } \\
\mathrm{g} \mathrm{cm}^{-3} \\
\end{array}$ & $\begin{array}{l}\text { Porosidade total } \\
\mathrm{cm} \mathrm{cm}^{-3} \\
\end{array}$ \\
\hline $100 \%$ SC 1 & 1,292 & 0,369 & 0,754 \\
\hline $20 \%$ LET $+80 \%$ SC 1 & 1,266 & 0,357 & 0,762 \\
\hline $40 \% \mathrm{LET}+60 \% \mathrm{SC} 1$ & 1,240 & 0,348 & 0,768 \\
\hline $60 \% \mathrm{LET}+40 \% \mathrm{SC} 1$ & 1,214 & 0,343 & 0,772 \\
\hline $80 \% \mathrm{LET}+20 \% \mathrm{SC} 1$ & 1,188 & 0,358 & 0,761 \\
\hline $100 \%$ LET & 1,158 & 0,347 & 0,769 \\
\hline $20 \% \mathrm{LET}+80 \% \mathrm{SC} 2$ & 0,836 & 0,119 & 0,921 \\
\hline $40 \% \mathrm{LET}+60 \% \mathrm{SC} 2$ & 0,915 & 0,208 & 0,861 \\
\hline $60 \% \mathrm{LET}+40 \% \mathrm{SC} 2$ & 0,995 & 0,275 & 0,817 \\
\hline $80 \%$ LET $+20 \% S C 2$ & 1,075 & 0,305 & 0,797 \\
\hline
\end{tabular}

Realizou-se a caracterização química para determinação dos teores totais de nutrientes nos diferentes substratos (Tabela 3 ) e a condutividade elétrica.

Tabela 3 - Caracterização química dos substratos formulados com diferentes proporções de lodo de esgoto tratado (LET) associado ao substrato à base de casca de pinus (SC1) ou substrato à base de fibra de coco (SC2), utilizados na produção de mudas de Plathymenia reticulata Benth.

Table 3 - Chemical characterization of substrates formulated with different proportions of sewage sludge treated (LET) with substrate based on pinus bark (SC1) or substrate based on coconut fiber (SC2) in the production of Plathymenia reticulata Benth seedlings.

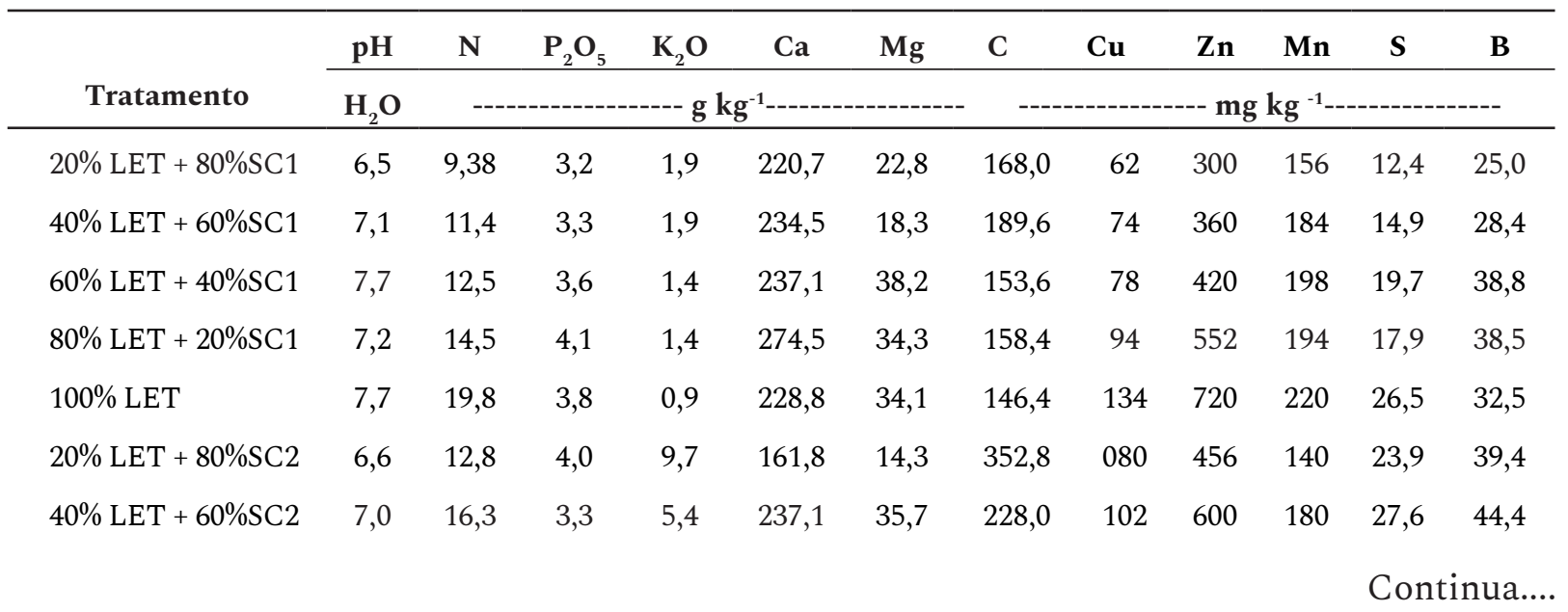


Tabela 3 - Conclusão...

Tabela 3 - Conclusion...

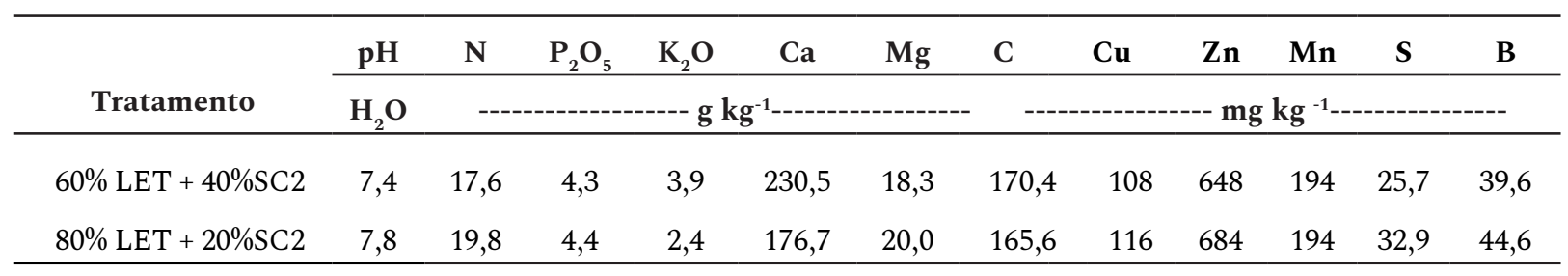

Análises realizadas no centro de análises pertencente à Universidade Federal Rural do Rio de Janeiro Campus de Campos dos Goytacazes utilizando-se metodologia proposta por EMBRAPA (2017).

Após 120 dias de crescimento na casa de vegetação, as mudas seguiram para a área de rustificação, permanecendo a pleno sol e com aumento gradual nos intervalos de aplicação de água. Nesta fase, as mudas foram molhadas até o escorrimento de água pelo orifício inferior do tubete, sendo que, em dias chuvosos, a aplicação de água foi suspensa. O procedimento foi feito manualmente, com auxílio de mangueiras, duas vezes ao dia, nos primeiros 10 dias e nos 10 dias finais da fase de rustificação, uma vez ao dia.

Após os 20 dias na fase de rustificação, avaliou-se a altura (H) e o diâmetro do coleto (DC) de todas as mudas. Foram selecionadas três mudas por parcela, com base no diâmetro médio para as análises destrutivas. Foi determinada a área foliar (AF), por meio do medidor de bancada (LICOR - LI3000).

Com relação à qualidade do torrão formado, avaliou-se a extração das mudas do tubete, analisada de acordo com a facilidade de retirada do recipiente sem danos à planta ou ao torrão, sendo atribuídas, pelo operador, notas de 1 a 5 , em que 1 representa a maior dificuldade de livrar a muda do recipiente e 5 , menor dificuldade (TRIGUEIRO, 2002).

A agregação do torrão foi avaliada de acordo com a quantidade de substrato aderida ao sistema radicular, sendo atribuídas notas de 1 a 5 , em que: nota 1 representa menos de $20 \%$ do substrato aderido ao sistema radicular, nota 2 , de 20 a $40 \%$, nota 3 , de 40 a $60 \%$, nota 4 , de 60 a $80 \%$ e nota 5 , de 80 a $100 \%$. Para determinar a porcentagem aderida, foi pesado o torrão (raiz + substrato) e o que restou no tubete. Deste valor, foi descontado o valor da massa fresca de cada raiz, pesada após a lavagem, sendo determinada assim, a porcentagem de substrato aderido ao sistema radicular por meio de regra de três simples (SIQUEIRA et al., 2018).

Avaliou-se a curvatura do torrão, atribuindo-se notas de 1 a 5 , de acordo com o grau de deformação ao ser colocado suspenso na horizontal. Classificou-se com nota 1 , a partir de $25^{\circ}$ de curvatura ou quando o torrão apresentou rachaduras ou quebra; nota 2 , de $20-25^{\circ}$; nota 3 , de $15-20^{\circ}$; nota 4 , de $10-15^{\circ}$ e nota 5 , de $5-10^{\circ}$ de curvatura (SIQUEIRA et al., 2018).

Após a avaliação da qualidade do torrão, o sistema radicular foi submetido ao processo de lavagem para retirada do substrato. A quantificação de raízes foi feita por meio do software WinRhizo, acoplado a um scanner, equipado com uma unidade de luz adicional, determinando-se o comprimento e o volume radicular com base em área de imagem $\left(8,4 \mathrm{~cm}^{2}\right)$, tomada aleatoriamente, gerada pelo software.

Avaliou-se a nodulação das mudas de acordo com a presença ou ausência de nódulos no sistema radicular das mudas, calculando-se a porcentagem de mudas com nódulos presentes. A massa seca da parte aérea (MSPA) e do sistema radicular (MSSR) das mudas foi determinada após secagem das amostras em estufa de circulação forçada de ar a $65^{\circ} \mathrm{C}$ por 72 horas.

Após a determinação da massa seca, os diferentes compartimentos foram moídos em moinho de facas, tipo Willey, e pesadas as amostras de $0,1 \mathrm{~g}$ em tubos de ensaio para determinação dos teores de nitrogênio $(\mathrm{N})$, fósforo $(\mathrm{P})$ e potássio $(\mathrm{K})$, e estimativa dos conteúdos. As amostras do material vegetal foram submetidas à oxidação por digestão sulfúrica $\left(\mathrm{H}_{2} \mathrm{O}_{4}\right.$ e $\left.\mathrm{H}_{2} \mathrm{O}_{2}\right)$ em sistema de digestão aberta. Em seguida, $\mathrm{o}$ $\mathrm{N}$ foi determinado por meio do método Nessler (JACKSON, 1965), o P pelo espectrofotômetro (Specord 
210) pelo método colorimétrico (azul de molibdato) e o K, determinado no fotômetro de emissão de chama.

As variáveis quantitativas foram submetidas à análise de variância e as diferenças a agrupamento de médias por meio do teste de Scott-Knott, a $5 \%$ de probabilidade. As variáveis qualitativas foram comparadas pelo teste não paramétrico de Kruskal-Wallis a $5 \%$ de probabilidade.

Embora os resultados não estejam apresentados neste trabalho, para comprovação da real qualidade das mudas produzidas nos diferentes substratos testados, foram selecionados os tratamentos que resultaram nas melhores mudas para avaliação do desempenho das mesmas a campo, analisando-se em longo prazo seu comportamento frente às condições adversas encontradas após plantio.

\section{Resultados e discussão}

Com relação aos atributos físicos dos substratos contendo LET e SC1, observou-se densidade de partícula compatível com a referência para materiais orgânicos - entre 1,2 e 1,5 $\mathrm{g} \mathrm{cm}^{-3}$ (PREDEVELLO, 1996). Quando as misturas foram feitas com SC2, a densidade ficou abaixo desta referência (Tabela 2), porém, tal fato também foi observado por Barreto e Testezlaf (2014). A maior porosidade total foi observada no substrato composto por $20 \%$ de LET e $80 \%$ de SC2, havendo redução à medida que se elevou a porcentagem de LET. A alta porosidade é característica da fibra de coco (CARRIJO; LIZ; MAKISHIMA, 2002; ZORZETO et al., 2014), podendo ser benéfica em condições de disponibilidade ótima de água, entretanto, em condição de deficit hídrico, a retenção de água pode ser prejudicada.

A caracterização química dos substratos testados (Tabela 3) revelou valores de pH e CE acima do sugerido como ideal, entre 5,0 e 5,8 (KAMPF, 2005) e de 1,0 $\mathrm{mS} \mathrm{cm}^{-1}$ (GONÇALVES et al., 2000), respectivamente. A CE do substrato contendo $100 \%$ de LET foi de $3,54 \mathrm{mS} \mathrm{cm}^{-1}$. Gonçalves et al. (2000) e Novais et al. (2007) destacam os efeitos deletérios do pH e CE altos, o que, no presente trabalho, pode ter influenciado o desenvolvimento das mudas de vinhático.

As mudas produzidas nos substratos contendo 20 ou $40 \%$ de LET em SC2 apresentaram altura $(\mathrm{H})$ inferior àquelas produzidas em substrato comercial fertilizado, no entanto, foram superiores às produzidas nos substratos com maiores quantidades de LET (Tabela 4). O diâmetro de colo (DC) das mudas produzidas em 20 e 40\% de LET foi semelhante àquelas produzidas em SC1 fertilizado e também superior às demais proporções testadas.

Resultados semelhantes foram verificados por Trigueiro e Guerrini (2014) em mudas de Schinus terebinthifolius, aos 120 dias após a semeadura, sendo observada redução de H e DC das mudas à medida que se elevou a quantidade de lodo em mistura com casca de arroz carbonizada. Os maiores valores foram observados em mudas produzidas em substrato comercial puro, contudo, a metodologia para tratamento do lodo não foi descrita pelos autores.

Em mudas de Enterolobium contortisiliquum, Teles, Costa e Gonçalves (1999) observaram redução em $\mathrm{H}$ e DC, produzidas em diferentes proporções de LET e terra de subsolo. Para higienização e estabilização do lodo, os autores também utilizaram a caleação, entretanto, a aplicação foi feita a $50 \%$ do peso seco do lodo, superior à utilizada neste trabalho. 
Tabela 4 - Altura (H), diâmetro do coleto (DC), área foliar (AF), massa seca da parte aérea (MSPA), massa seca do sistema radicular (MSSR) e relação matéria seca do sistema radicular/massa seca da parte aérea (MSSR/MSPA) de mudas de Plathymenia reticulata Benth produzidas em diferentes substratos, aos 140 dias após a semeadura.

Table 4 - Height (H), stem diameter (DC), leaf area (AF), shoot dry mass (MSPA), root dry mass (MSSR) and root dry mass/shoot ratio (MSSR/MSPA) in Plathymenia reticulata Benth seedlings produced in different substrates, 140 days after sowing.

\begin{tabular}{|c|c|c|c|c|c|c|}
\hline Tratamento & $\begin{array}{c}\mathrm{H} \\
\mathrm{cm}\end{array}$ & $\begin{array}{l}\mathrm{DC} \\
\mathrm{mm}\end{array}$ & $\begin{array}{l}\mathrm{AF} \\
\mathrm{cm}^{2}\end{array}$ & MSPA & MSSR & MSSR/MSPA \\
\hline $\mathrm{SC} 1+\mathrm{A}$ & $26,77 \mathrm{~A}^{1}$ & $4,17 \mathrm{~A}$ & $224,8 \mathrm{~A}$ & $2,10 \mathrm{~A}$ & $1,12 \mathrm{~A}$ & $0,54 \mathrm{~A}$ \\
\hline $20 \%$ LET $+80 \%$ SC 1 & $21,88 \mathrm{~B}$ & $3,71 \mathrm{~A}$ & $211,8 \mathrm{~A}$ & $1,83 \mathrm{~A}$ & $0,87 \mathrm{~A}$ & $0,47 \mathrm{~A}$ \\
\hline $40 \%$ LET $+60 \%$ SC1 & $18,50 \mathrm{C}$ & $3,15 \mathrm{~A}$ & $151,6 \mathrm{~A}$ & $1,49 \mathrm{~A}$ & $0,59 \mathrm{~B}$ & $0,41 \mathrm{~A}$ \\
\hline $60 \%$ LET + $40 \%$ SC 1 & $14,86 \mathrm{C}$ & $2,18 \mathrm{~B}$ & 84,18 B & $0,80 \mathrm{~B}$ & $0,30 \mathrm{C}$ & $0,39 \mathrm{~A}$ \\
\hline $80 \%$ LET $+20 \%$ SC 1 & $14,80 \mathrm{C}$ & 2,43 B & $108,2 \mathrm{~B}$ & $0,94 \mathrm{~B}$ & $0,34 \mathrm{C}$ & $0,44 \mathrm{~A}$ \\
\hline $100 \% \mathrm{LET}$ & $15,58 \mathrm{C}$ & $2,50 \mathrm{~B}$ & $93,20 \mathrm{~B}$ & $0,87 \mathrm{~B}$ & $0,29 \mathrm{C}$ & $0,43 \mathrm{~A}$ \\
\hline $20 \%$ LET $+80 \%$ SC2 & $20,29 \mathrm{~B}$ & $3,56 \mathrm{~A}$ & $180,0 \mathrm{~A}$ & $1,15 \mathrm{~A}$ & $0,62 \mathrm{~B}$ & $0,59 \mathrm{~A}$ \\
\hline $40 \%$ LET $+60 \%$ SC 2 & 20,16 B & $3,50 \mathrm{~A}$ & $204,8 \mathrm{~A}$ & $1,58 \mathrm{~A}$ & 0,63 B & $0,42 \mathrm{~A}$ \\
\hline $60 \%$ LET $+40 \%$ SC 2 & $18,34 \mathrm{C}$ & $3,01 \mathrm{~B}$ & $180,0 \mathrm{~A}$ & $1,55 \mathrm{~A}$ & $0,60 \mathrm{~B}$ & $0,41 \mathrm{~A}$ \\
\hline $80 \%$ LET $+20 \%$ SC 2 & $14,70 \mathrm{C}$ & $2,52 \mathrm{~B}$ & $75,60 \mathrm{~B}$ & 0,61 B & $0,24 \mathrm{C}$ & $0,42 \mathrm{~A}$ \\
\hline CV (\%) & 14,08 & 15,31 & 34,39 & 38,89 & 37,20 & 45,24 \\
\hline
\end{tabular}

${ }^{1}$ Médias seguidas de letras iguais, na coluna, não diferem entre si pelo teste de Scott Knott, em $5 \%$ de significância. Em que: LET = Lodo de esgoto tratado; SC1 = Substrato comercial à base de casca de pinus; $\mathrm{A}=$ adubo de liberação lenta e SC2 = substrato comercial à base de fibra de coco.

Delarmelina et al. (2014) encontraram a maior altura de mudas de Sesbania virgata produzidas em $60 \%$ de LET em vermiculita, entretanto, para tratamento do lodo, os autores utilizaram a exposição do resíduo ao sol, não promovendo alcalinidade do mesmo.

De acordo com Gonçalves et al. (2000), mudas de espécies nativas de boa qualidade para plantio devem possuir altura entre 20 e $35 \mathrm{~cm}$, assim, os tratamentos: SC1 fertilizado, 20\% em ambas as misturas e $40 \%$ de LET em SC2 produziram mudas que atenderam aos requisitos mínimos sugeridos pelo autor.

A redução no crescimento aéreo das mudas nas maiores proporções de LET também resultou em menor área foliar (AF), massa seca da parte aérea (MSPA) e do sistema radicular (MSSR). A formação de mudas com menor AF pode ser benéfica em sítios de plantio com elevada temperatura e/ou baixa pluviosidade, reduzindo perdas de água e favorecendo a sobrevivência das mudas após o plantio. Forrester (2013) destaca que, a redução da parte aérea das plantas resulta em menor captação de recursos por meio da fotossíntese, mas que, no entanto, algumas vezes tal redução pode ser vantajosa pela compensação com o aumento da eficiência do remanescente foliar, sendo capaz de suprir a demanda fotossintética.

Gomes e Paiva (2011) ressal tam que a sobrevivência, o estabelecimento e a boa produtividade dos povoamentos florestais são consideravelmente maiores quanto mais abundante for o sistema radicular, independente da altura da parte aérea, podendo ser representado pela MSSR, o que indica que, possivelmente, as mudas produzidas no substrato comercial fertilizado e na proporção de $20 \%$ de LET associado ao SC1 podem ter maior probabilidade de sobrevivência pós-plantio. 
Trazzi et al. (2014), avaliando o LET como substrato para produção de mudas de Tectona grandis encontraram a maior MSSR no substrato contendo $60 \%$ de LET $+40 \%$ de fibra de coco, proporção de LET superior à observada neste trabalho. Os mesmos autores afirmam que a mistura entre LET e fibra de coco confere boa interação entre as características físicas, promovendo adequada aeração do sistema radicular, entretanto, não foram descritas pelos autores as características do torrão formado.

Quando avaliada a relação entre a massa seca do sistema radicular/massa seca da parte aérea (MSSR/MSPA), embora sem diferença estatística, as mudas produzidas em SC1 fertilizado ou quanto utilizada a menor proporção de LET em SC2 apresentaram valores mais próximos ao sugerido como ideal, que é de 0,5 . Este valor reflete em um bom padrão de qualidade das mudas, pressupondo melhor alocação da biomassa entre os compartimentos (DANIEL et al., 1997).

Com relação à qualidade do torrão formado, pouco abordada na literatura, a extração da muda do tubete foi ligeiramente facilitada nas misturas feitas com a SC2 em relação àquelas utilizando o SC1 (Tabela 5). Possivelmente, este fato ocorreu devido às melhores características físicas apresentadas por essas formulações de substrato, tendo em vista a possibilidade das raízes se enovelarem nas fibras, melhorando a qualidade do torrão. Gomes e Paiva (2011) classificam a agregação do sistema radicular ao substrato como um dos requisitos para a determinação da qualidade das mudas. Para o presente estudo, a agregação não foi afetada pela adição de LET ao substrato.

O SC1 fertilizado e a proporção de $20 \%$ em ambas as misturas e $40 \%$ de LET em SC2 apresentaram a menor curvatura, indicando que os torrões destes tratamentos mantiveram alta estabilidade quando posicionados suspensos na horizontal, favorecendo o processo de plantio, especialmente mecanizados.

As maiores curvaturas do torrão foram verificadas nas mudas produzidas em substratos com maiores proporções de LET, mesmos tratamentos que apresentaram redução na MSSR (Tabela 4), comprimento e volume radicular, evidenciando os danos ao enraizamento das mudas e seu efeito sobre a qualidade final do torrão formado.

Trigueiro e Guerrini (2003) também observaram que a adição de LET ao substrato influenciou negativamente a extração de mudas de eucalipto dos tubetes, o que atribuíram ao baixo enraizamento apresentado nas maiores proporções de LET, corroborando este trabalho.

Com relação à curvatura do torrão, Siqueira et al. (2018), avaliando diferentes combinações de LET e substrato comercial à base de casca de pinus para produção de mudas de Lafoensia glyptocarpa, concluíram que, a partir de $50 \%$ de LET, houve aumento da curvatura do torrão formado, mesmo comportamento deste trabalho nas maiores proporções de LET. Além disso, Cabreira et al. (2017) destacam que, quando utilizado o lodo de esgoto na composição de substratos para produção de mudas florestais, o peso das mudas formadas é diminuído, facilitando a logística de expedição para o campo.

As mudas produzidas nos substratos contendo $20 \%$ de LET em SC1 e até $40 \%$ quando em SC2 apresentaram mesmo comprimento e volume radicular daquelas produzidas em substrato comercial adubado. As misturas de LET e SC2 podem ter sido levemente favorecidas pela menor densidade e maior porosidade apresentada, melhorando a aeração do sistema radicular. Trigueiro e Guerrini (2014) observaram o maior desenvolvimento radicular de mudas de Schinus terebinthifolius produzidas em substrato comercial adubado em relação às produzidas em diferentes proporções de lodo de esgoto, atribuindo este resultado às características físicas apresentadas pelo resíduo. 
Tabela 5 - Comprimento e volume de raiz, porcentagem de mudas com presença de nódulos radiculares (Nodulação) e pontuação qualitativa dada à facilidade de extração, agregação e curvatura do torrão de mudas de Plathymenia reticulata Benth produzidas em diferentes substratos, aos 140 dias após a semeadura.

Table 5 - Root lenght, root volume, percentage of seedlings with root nodules and qualitative score given for extraction facility, aggregation and curvature of clod in Plathymenia reticulata

Benth seedlings produced in different substrates, 140 days after sowing.

\begin{tabular}{|c|c|c|c|c|c|c|}
\hline Tratamento & $\begin{array}{c}\text { Comprimento } \\
\text { de raiz } \\
\mathrm{cm} \\
\end{array}$ & $\begin{array}{c}\text { Volume de } \\
\text { raiz } \\
\mathrm{cm}^{2} \\
\end{array}$ & $\begin{array}{c}\text { Nodulação } \\
\% \\
\end{array}$ & Extração & Agregação & Curvatura \\
\hline $\mathrm{SC} 1+\mathrm{A}$ & $89,94 \mathrm{~A}^{1}$ & $0,31 \mathrm{~A}$ & $100 \mathrm{~A}$ & $4,54 \mathrm{~B}^{2}$ & $5,00 \mathrm{~A}$ & $5,00 \mathrm{~A}$ \\
\hline $20 \%$ LET $+80 \%$ SC 1 & $95,08 \mathrm{~A}$ & $0,23 \mathrm{~A}$ & $55 \mathrm{~A}$ & $4,41 \mathrm{~B}$ & $5,00 \mathrm{~A}$ & $4,35 \mathrm{~A}$ \\
\hline $40 \%$ LET $+60 \%$ SC 1 & $57,28 \mathrm{~B}$ & $0,15 \mathrm{~B}$ & $33 \mathrm{~B}$ & $4,67 \mathrm{~B}$ & $4,67 \mathrm{~A}$ & $3,33 \mathrm{~B}$ \\
\hline $60 \% \mathrm{LET}+40 \% \mathrm{SC} 1$ & $36,40 \mathrm{~B}$ & $0,09 \mathrm{~B}$ & $11,3 \mathrm{~B}$ & 4,67 B & $4,08 \mathrm{~A}$ & $1,91 \mathrm{C}$ \\
\hline $80 \% \mathrm{LET}+20 \% \mathrm{SC} 1$ & $37,05 \mathrm{~B}$ & $0,09 \mathrm{~B}$ & $0 \mathrm{~B}$ & $4,91 \mathrm{~A}$ & $4,67 \mathrm{~A}$ & $2,25 \mathrm{C}$ \\
\hline $100 \% \mathrm{LE}$ & $33,90 \mathrm{~B}$ & $0,08 \mathrm{~B}$ & $0 \mathrm{~B}$ & $4,67 \mathrm{~B}$ & $4,75 \mathrm{~A}$ & $1,50 \mathrm{C}$ \\
\hline $20 \% \mathrm{LET}+80 \%$ SC2 & $97,98 \mathrm{~A}$ & $0,26 \mathrm{~A}$ & $77,3 \mathrm{~A}$ & $5,00 \mathrm{~A}$ & $5,00 \mathrm{~A}$ & $5,00 \mathrm{~A}$ \\
\hline $40 \%$ LET $+60 \%$ SC2 & $74,07 \mathrm{~A}$ & $0,18 \mathrm{~A}$ & $33 \mathrm{~B}$ & $4,91 \mathrm{~A}$ & $5,00 \mathrm{~A}$ & $4,67 \mathrm{~A}$ \\
\hline $60 \%$ LET $+40 \%$ SC 2 & $42,50 \mathrm{~B}$ & $0,11 \mathrm{~B}$ & $0 \mathrm{~B}$ & $5,00 \mathrm{~A}$ & $5,00 \mathrm{~A}$ & $3,66 \mathrm{~B}$ \\
\hline $80 \%$ LET $+20 \%$ SC2 & $94,78 \mathrm{~B}$ & $0,08 \mathrm{~B}$ & $11,3 \mathrm{~B}$ & $4,91 \mathrm{~A}$ & $4,67 \mathrm{~A}$ & $1,50 \mathrm{C}$ \\
\hline CV $(\%)$ & 29,26 & 36,45 & 82,47 & 5,06 & 8,95 & 23,78 \\
\hline
\end{tabular}

${ }^{1}$ Médias seguidas de letras iguais, na coluna, não diferem entre si pelo teste de Scott Knott, em $5 \%$ de significância. ${ }^{2}$ Médias seguidas de letras iguais, na coluna, não diferem entre si pelo teste de KruskalWallis em 5\% de significância. Em que: LET = Lodo de esgoto tratado; SC1 = Substrato comercial à base de casca de pinus; $\mathrm{A}$ = adubo de liberação lenta e SC2 = substrato comercial à base de fibra de coco.

A adição de $20 \%$ e $40 \%$ de LET ao SC2 resultou em mudas com menor MSSR, no entanto, o comprimento radicular destas mudas não diferiu daquelas produzidas em SC1 fertilizado. Tal fato sugere a formação de maior quantidade de raízes finas, e, consequentemente, mais ativas em tais formulações de substrato.

SC1 fertilizado e a proporção de $20 \%$ de LET em ambas misturas resultaram em mudas com maior percentual de nódulos radiculares presentes, resultantes da simbiose com bactérias diazotróficas, quando comparados aos demais substratos testados. A utilização de LET e seus efeitos sobre a nodulação de mudas florestais são pouco conhecidos, especialmente para espécies nativas. Para o presente trabalho, a menor nodulação das mudas nas maiores proporções de LET pode ter sido ocasionada pelos altos valores de pH (ARANJUELO; ARRESE-IGOR; MOLERO, 2014) e pela presença de metais pesados (TRANNIN; MOREIRA; SIQUEIRA, 2001), contudo, a nodulação não influenciou o conteúdo de nitrogênio $(\mathrm{N})$ na parte aérea (Tabela 6).

Os conteúdos de fósforo $(\mathrm{P})$ e potássio $(\mathrm{K})$ foram maiores nas mudas produzidas em SC1 adubado, com redução mais acentuada nas mudas produzidas em substrato com proporções a partir de $40 \%$ de LET. Não houve variação do K entre as mudas produzidas nos substratos com diferentes proporções de LET e SC2. Apesar do menor conteúdo de $\mathrm{P}$ e K, as mudas produzidas nas menores proporções de LET não apresentaram variações biométricas em relação à testemunha, evidenciando maior eficiência de utilização destes nutrientes pelas mudas. 
Trazzi (2011) encontrou maior conteúdo de N, P e K em mudas de Tectona grandis produzidas em substratos que receberam LET, sendo este resultado atribuído à maior disponibilidade destes nutrientes, entretanto, os autores não utilizaram qualquer tipo de adubação no tratamento contendo substrato comercial puro, o que desfavorece o mesmo.

Composto à base de lodo de esgoto e poda de árvores trituradas em diferentes proporções (3:1 e 2:1, v:v) e três níveis de fertilizantes mineral granulado $(0 ; 2,7$ e 4,0 g dm-3) testados por Scheer, Bressan e Santos (2012) para produção de mudas de Lafoensia pacari, geraram mudas com maiores conteúdos de $\mathrm{N}$ e $\mathrm{P}$ na biomassa aérea daquelas produzidas nas maiores proporções do composto à base de lodo e nas maiores doses de fertilizantes, em relação ao substrato comercial.

\section{Tabela 6 - Conteúdo de N, P e K na biomassa aérea e radicular de mudas de Plathymenia reticulata Benth produzidas em diferentes substratos, aos 140 dias após a semeadura.}

Table $6-\mathrm{N}, \mathrm{P}$ and $\mathrm{K}$ content in leaf and root biomass in Plathymenia reticulata Benth seedlings produced in different substrates, 140 days after sowing.

\begin{tabular}{|c|c|c|c|c|c|c|}
\hline Tratamento & $\mathbf{N}$ pa & $\mathrm{N} \mathrm{r}$ & P pa & $\begin{array}{r}\mathbf{P r} \\
\text { anta }^{-1} \\
\end{array}$ & K pa & $\mathbf{K r}$ \\
\hline $\mathrm{SC} 1+\mathrm{A}$ & $16,1 \mathrm{~A}^{1}$ & $4,4 \mathrm{~B}$ & $6,44 \mathrm{~A}$ & $4,29 \mathrm{~A}$ & $21,7 \mathrm{~A}$ & $16,9 \mathrm{~A}$ \\
\hline $20 \%$ LET $+80 \%$ SC1 & $20,6 \mathrm{~A}$ & $6,1 \mathrm{~B}$ & $3,66 \mathrm{~B}$ & $2,46 \mathrm{~B}$ & $14,4 \mathrm{~B}$ & $7,51 \mathrm{~B}$ \\
\hline $40 \%$ LET $+60 \%$ SC 1 & $23,5 \mathrm{~A}$ & $6,2 \mathrm{~B}$ & $1,16 \mathrm{C}$ & $1,41 \mathrm{C}$ & $10,7 \mathrm{C}$ & $5,90 \mathrm{~B}$ \\
\hline $60 \% \mathrm{LET}+40 \% \mathrm{SC} 1$ & $24,7 \mathrm{~A}$ & $7,5 \mathrm{~B}$ & $0,44 \mathrm{C}$ & $0,60 \mathrm{C}$ & $6,70 \mathrm{C}$ & $2,93 \mathrm{C}$ \\
\hline $80 \%$ LET $+20 \%$ SC1 & $24,8 \mathrm{~A}$ & $9,7 \mathrm{~A}$ & $0,49 \mathrm{C}$ & $0,98 \mathrm{C}$ & $7,30 \mathrm{C}$ & $2,30 \mathrm{C}$ \\
\hline $100 \% \mathrm{LE}$ & $30,2 \mathrm{~A}$ & $10,8 \mathrm{~A}$ & $0,42 \mathrm{C}$ & $0,70 \mathrm{C}$ & $6,80 \mathrm{C}$ & $2,18 \mathrm{C}$ \\
\hline $20 \% \mathrm{LET}+80 \%$ SC2 & $31,7 \mathrm{~A}$ & $10,9 \mathrm{~A}$ & $3,70 \mathrm{~B}$ & $2,27 \mathrm{~B}$ & $7,50 \mathrm{C}$ & $2,98 \mathrm{C}$ \\
\hline $40 \%$ LET $+60 \%$ SC 2 & $35,3 \mathrm{~A}$ & $11,0 \mathrm{~A}$ & $2,67 \mathrm{~B}$ & $2,04 \mathrm{~B}$ & $10,1 \mathrm{C}$ & $2,77 \mathrm{C}$ \\
\hline $60 \%$ LET $+40 \%$ SC2 & $35,8 \mathrm{~A}$ & $11,8 \mathrm{~A}$ & $1,03 \mathrm{C}$ & $1,75 \mathrm{C}$ & $10,0 \mathrm{C}$ & $2,60 \mathrm{C}$ \\
\hline $80 \%$ LET + 20SC2 & $46,1 \mathrm{~A}$ & $16,3 \mathrm{~A}$ & $0,30 \mathrm{C}$ & $0,59 \mathrm{C}$ & $5,21 \mathrm{C}$ & $1,53 \mathrm{C}$ \\
\hline CV $(\%)$ & 41,15 & 41,36 & 63 & 45,70 & 33,08 & 46,89 \\
\hline
\end{tabular}

${ }^{1}$ Médias seguidas de letras iguais, na coluna, não diferem entre si pelo teste de Scott Knott, em $5 \%$ de significância. Em que: LET = Lodo de esgoto tratado; SC1 = Substrato comercial à base de casca de pinus; $\mathrm{A}$ = adubo de liberação lenta e SC2 = substrato comercial à base de fibra de coco.

O maior acúmulo de K em mudas de Schinus terebinthifolius foi observado por Trigueiro e Guerrini (2014) na utilização do substrato comumente utilizado nos viveiros, em relação a diferentes proporções de LET testadas, como observado no presente trabalho. Abreu et al. (2017) também trabalhando com mudas de Schinus terebinthifolius observaram aumento no teor de nutrientes, principalmente N, P e K, à medida que se elevou a proporção de lodo de esgoto no substrato.

Cunha et al. (2006), também observaram maior acúmulo de $\mathrm{N}$ nas mudas de Acacia sp. produzidas na maior proporção de LET (100\%), principalmente quando inoculadas com rizóbios, no entanto, estes tratamentos apresentaram maior crescimento de mudas, contrário ao observado neste trabalho para o mesmo tratamento. Além disso, não foi descrita pelos autores a metodologia para tratamento do lodo nem as características do torrão formado.

Não houve grande variação nos teores totais de $\mathrm{P}_{2} \mathrm{O}_{5}$ e $\mathrm{K}_{2} \mathrm{O}$ verificados nas diferentes composições de substrato (Tabela 3), contudo, o mesmo não foi observado nos conteúdos destes nutrientes, o que possivelmente foi ocasionado pelos altos valores de $\mathrm{pH}$, alterando a 
disponibilidade dos nutrientes. Além disso, a alta CE pode ter afetado negativamente o sistema radicular (KAMPF, 2005), ocasionando problemas para a absorção dos nutrientes.

\section{Conclusões}

A menor proporção de lodo de esgoto em ambas misturas se mostrou a mais adequada para produção de mudas de Plathymenia reticulata Benth, com padrão de crescimento semelhante àquelas produzidas em substrato comercial à base de casca de pinus fertilizado.

A agregação do torrão ao substrato não foi influenciada pela adição de LET ao substrato, no entanto, a curvatura foi prejudicada nas maiores proporções de LET.

\section{Referências}

ABREU, A. H. M. et al. Caracterização e potencial de substratos formulados com biossólido na produção de mudas de Schinus terebinthifolius Raddi. e Handroanthus heptaphyllus (Vell.) Mattos. Ciência Florestal, Santa Maria, v. 27, n. 4, p. 1179-1190, 2017.

ARANJUELO, I.; ARRESE-IGOR, C.; MOLERO, G. Nodule performance within a changing environmental context. Journal of Plant Physiology, Minneapolis, v. 171, p. 1076-1090, 2014.

BARRETO, C. V. G.; TESTEZLAF, R. Particle size distribuition effects on physical characteristics of coconut and pine bark substrates. Revista de Ciências Agroveterinárias, Lages, v. 13, n. 3, p. 327-336, 2014.

BRASIL. Ministério do Meio Ambiente. Conselho Nacional do Meio Ambiente. Resolução n. 375/2006. Define critérios e procedimentos para o uso agrícola de lodos de esgoto gerados em estações de tratamento de esgoto sanitário e seus produtos derivados. Diário Oficial da República Federativa do Brasil, Brasília, n. 167, p. 141-146, 30 ago. 2006.

CABREIRA, G. V. et al. Biossólido como componente de substrato para produção de mudas florestais. Floresta, Curitiba, v. 47, n. 2, p. 165-176, 2017.

CALDEIRA, M. V, W. et al. Substratos alternativos na produção de mudas de Chamaecrista desvauxii. Revista Árvore, Viçosa, MG, v. 37, n. 1, p. 31-39, 2013.

CARRIJO, O. A.; LIZ, R. S.; MAKISHIMA, N. Fibra de coco verde como substrato agrícola. Horticultura Brasileira, Vitória da Conquista, v. 20, n. 4, p. 533-535, 2002.

CARVALHO, P. E. R. Espécies arbóreas brasileiras. Brasília: Embrapa Informação Tecnológica; Colombo: Embrapa Florestas, 2010. v. 4. 644 p. (Coleção Espécies Arbóreas Brasileiras).

CUNHA, A. M. et al. Efeito de diferentes substratos sobre o desenvolvimento de mudas de Acacia sp. Revista Árvore, Viçosa, MG, v. 30, n. 2, p. 207-214, 2006.

DANIEL, O. et al. Aplicação de fósforo em mudas de Acacia mangium. Revista Árvore, Viçosa, MG, v. 21, n. 2, p. 163-168, 1997.

DELARMELINA, W. M. et al. Diferentes substratos para produção de mudas de Sesbania virgata. Floresta e Ambiente, Seropédica, v. 21, n. 2, p. 224-233, 2014.

FORRESTER, D. I. Growth responses to thinning, prunning and fertiliser application in Eucalyptus plantations: A review of their production ecology and interactions. Forest Ecology and Management, Amsterdam, v. 310, p. 336-347, 2013.

EMBRAPA. Manual de métodos de análises de solo. 3. ed. Brasília, 2017. 575 p.

GOMES, D. R. et al. Lodo de esgoto como substrato para produção de mudas de Tectona grandis. Cerne, Lavras, v. 19, n. 1, p. 123-131, 2013.

GOMES, J. M.; PAIVA, H. N. Viveiros florestais: propagação sexuada. Viçosa, MG: UFV, 2011. $116 \mathrm{p}$.

GONÇALVES, J. L. M. et al. Produção de mudas de espécies nativas: substrato, nutrição, sombreamento e fertilização. In: GONÇALVES, J. L. M.; BENEDETTI, V. (Ed.). Nutrição e fertilização florestal. Piracicaba: IPEF, 2000. p. 309-350. 
Siqueira, D. P.; Barroso, D. G.; Carvalho, G. C. M. W.;

Erthal, R. M.; Rodrigues, M. C. C.; Marciano, C. R.

JACKSON, M. L. Soil chemical analysis. New Jersey: Prentice Hall, 1965. 498 p.

KAMPF, A. N. Produção comercial de plantas ornamentais. Guaíba: Agropecuária, 2005. 256 p.

LACERDA, D. R. et al. A técnica RAPD: uma ferramenta molecular em estudos de conservação de plantas. Lundiana, Belo Horizonte, v. 3, n. 2, p. 87-92, 2002.

LOUSADA, L. L. Nutrição e crescimento do sorgo sacarino e alterações nos atributos do solo pela aplicação de lodo de esgoto doméstico. 2015. 122 f. Tese (Doutorado em Produção Vegetal) - Universidade Estadual do Norte Fluminense, Campos dos Goytacazes, 2015.

NOVAIS, R. F. et al. Fertilidade do solo. Viçosa, MG: Sociedade Brasileira de Ciência do Solo, 2007. 1017 p.

PREVEDELLO, C. L. Física do solo com problemas resolvidos. Curitiba: [s.n.], 1996. 446 p.

ROCHA, J. H. T. et al. Composto de lodo de esgoto como substrato para produção de mudas de eucalipto. Pesquisa Florestal Brasileira, Colombo, v. 33, n. 73, p. 27-36, 2013.

SANTOS, E. V. F. et al. Características químicas de substratos formulados com lodo de esgoto para produção de mudas florestais. Revista Brasileira de Engenharia Agrícola e Ambiental, Campina Grande, v. 18, n. 9, p. 971-979, 2014.

SIQUEIRA, D. P. et al. Lodo de esgoto tratado na composição de substrato para produção de mudas de Lafoensia glyptocarpa. Floresta, Curitiba, v. 48, n. 2, p. 277-284, 2018.

SCHEER, M. B.; BRESSAN, C. C. O. A.; SANTOS, K. G. Crescimento e nutrição de mudas de Lafoensia pacari com lodo de esgoto. Floresta e Ambiente, Seropédica, v. 19, n. 1, p. 55-65, 2012.

TELES, C. R.; COSTA, A. N.; GONÇALVES, R. F. Produção de lodo em lagoas de estabilização e seu uso no cultivo de espécies florestais na região Sudoeste do Brasil. Sanare, Vitória, v. 12, n. 12, p. 53-60, 1999.

TRANNIN, I. C. B.; MOREIRA, F. M. S.; SIQUEIRA, J. O. Crescimento e nodulação de Acacia mangium, Enterolobium contortisiliquum e Sesbania virgata em solo contaminado com metais pesados. Revista Brasileira de Ciências do Solo, Viçosa, MG, v. 25, n. 1, p. 743-753, 2001.

TRAZZI, P. A. Substratos renováveis na produção de mudas de Tectona grandis Linn F. 2011. 98 f. Dissertação (Mestrado em Ciências Florestais) - Universidade Federal do Espirito Santo, Jerônimo Monteiro, 2011.

TRAZZI, P. A. et al. Produção de mudas de Tectona grandis em substratos formulados com biossólido. Cerne, Lavras, v. 20, n. 2, p. 293-302, 2014.

TRIGUEIRO, R. M. Uso de biossólido como substrato para produção de mudas de pinus e eucalipto. 2002. 94 f. Dissertação (Mestrado em Agronomia) - Universidade Estadual Paulista "Júlio de Mesquita Filho", Botucatu, 2002.

TRIGUEIRO, R. M.; GUERRINI, I. A. Uso de biossólido como substrato para produção de mudas de eucalipto. Scientia Forestalis, Piracicaba, n. 64, p. 150-162, 2003.

TRIGUEIRO, R. M.; GUERRINI, I. A. Utilização de lodo de esgoto na produção de mudas de aroeira-pimenteira. Revista Árvore, Viçosa, MG', v. 38, n. 4, p. 657-665, 2014.

ZORZETO, T. Q. et al. Caracterização Campinas, v. 73, n. 3, p. 300-311, 2014.

física de substratos para plantas. Bragantia, 